\title{
Efficient Organization of Data Center for Cloud Computing: A Survey
}

\author{
Md. Abdullah-Al-Shafi ${ }^{1 *}$ and Ali Newaz Bahar ${ }^{2}$ \\ ${ }^{1}$ Institute of Information Technology (IIT), University of Dhaka, \\ Dhaka, Bangladesh \\ ${ }^{2}$ Department of ICT, Mawlana Bhashani Science and Technology University, \\ Tangail, Bangladesh \\ alshafi08@gmail.com
}

\begin{abstract}
Today, data centers power utilization has massive influences on environments. Data centers are energy-starved, crucial structures that direct big-scale internet-based facilities. The extreme energy utilization and green contamination of data centers have turned into a serious concern. Energy expenditure archetypes are decisive in planning and improving energy-resourceful functions to control extreme energy utilization in data center. Experts are looking for locating efficient explanations to build data centers diminish energy expenditure whereas retaining the preferred feature of service objectives. Hence, GreenCloud or Internet-based processing answers are desired that cannot merely lessen operating expenses but also prevent energy for the natural environment. This study organizes architectural foundations, resource distribution for data Centre and challenges for energy proficient organization of cloud computing atmospheres. Besides energyeconomy fashions for data centers in future are presented in this paper.
\end{abstract}

Keywords: Data Centre, Energy efficacy, Cloud computing, Energy preservation

\section{Introduction}

Data centers are identified as warehouses of a computer which keep a huge volume of records for separate associations so that form their regular transaction handling necessities [1]. It may possibly be believed as an assembly of separate network organizations and servers, where servers are operated to assemble records, network organizations are operated to retain, utilize and inform server's records and consumers can approach the records center servers through the network. In recent times, cloud computing [2] has fascinated extensive and it is considered to be one of the supreme imperative imminent computing and facility archetype [3]. A cloud is a sort of circulated and parallel organization involving an assembly of virtualized and connected computers which are actively performed as unique or more combined computing sources established on provision-level arrangements started through compromise between the facility supplier and clients [4]. In that way, consumers will be capable of approaching applications and records from a "cloud" anyplace around the planet on demand. The cloud seems to be a particular fact of approach for all the computing demands of clients [5]. The data center is a frequent system to present cloud computing. An internet data center generally arranges lots of servers, compactly filled to expand the area of deployment. Continuing facilities in combined servers in data center offer consumers a substitute to proceeding the software or managing the computer facilities in-house. Table 1 presents a concise view of server power deployment modeling procedures. The foremost advantages of data center

Received (January 20, 2018), Review Result (May 2, 2018), Accepted (May 13, 2018)

* Corresponding Author 
comprise the practice of economies of the level to repay the charge of control and the charge of maintenance throughout a huge volume of machines. With the swift progress of internet data center, the energy utilized by the data center, openly interrelated to the amount of offered servers and their capacity has been rise steeply [6]. The esteemed power utilizations of servers have enlarged by 10 times over the earlier ten years [7]. This rushing requirement calls for the persuasive requirement of devising and arrangement of energy-competent data centers. GreenCloud is a data center structure that directs to diminish data center power utilization, whereas simultaneously assurance the operation from client's viewpoint. A huge dispute for GreenCloud is to routinely prepare the organizing resolution on actively consolidating virtual machines among servers to collect the capacity conditions in the meantime keeping energy, notably for performingresponsive functions, for example, live gaming servers. The key purpose of this study is to introduce research and progress of energy-responsive resource provision systems and records for data centers in order to cloud computing can be a more maintainable environmental technology to handle profitable, technical, and scientific improvement for next generations.

Table 1. Review of Server Power Utilization Modeling Methods

\begin{tabular}{|c|l|l|}
\hline Works & \multicolumn{1}{|c|}{ Qualities } & \multicolumn{1}{|c|}{ Energy efficacy } \\
\hline In [21] & $\begin{array}{l}\text { Picking per-loop processor } \\
\text { clock frequencies }\end{array}$ & $\begin{array}{l}\text { Capable of 7.6\% on an 8-core Intel } \\
\text { Xeon E5530 and 10.6\% on a 32-core } \\
\text { AMD Opteron 8380 }\end{array}$ \\
\hline In [22] & $\begin{array}{l}\text { Considerably refining the } \\
\text { usage effectivenes of log } \\
\text { pages by eradicating auto-date } \\
\text { log archives }\end{array}$ & $\begin{array}{l}\text { Considerably progress the } \\
\text { performance, energy ingestion, and } \\
\text { lifetime of the NAND flash memory } \\
\text { storage }\end{array}$ \\
\hline In [23] $\begin{array}{l}\text { The data separating appliance } \\
\text { permitting DCAPS to modify } \\
\text { the parallelism degrees of write } \\
\text { requests }\end{array}$ & $\begin{array}{l}\text { Up to 70\% energy reserves } \\
\text { Expecting sub-blocks of a } \\
\text { eache line that will be } \\
\text { In [24 }\end{array}$ & $\begin{array}{l}\text { Almost 24\% energy reduction for the } \\
\text { whole cache hierarchy }\end{array}$ \\
\hline In [25] $\begin{array}{l}\text { Expending dynamic profiling } \\
\text { to estimate the memory } \\
\text { performance energy and }\end{array}$ & $\begin{array}{l}\text { The average saving by using Cashier } \\
\text { being 23.6\% }\end{array}$ \\
\hline
\end{tabular}

\section{Architectural Fundamentals of GreenCloud}

The purpose of this analysis is to focus the challenge of allowing energy-competent resource provision, so directing to green computing data centers, to assure participating application's requirement for computing service area and recover energy. Figure 1 presents the approach for maintaining energy-competent assistance provision in green computing. The fundamental units are organized below.

a) Physical Engines: The contributory base servers deliver hardware foundation for generating virtualized sources to organize provision requirements.

b) Virtual Engines: Several virtual engines can actively begin and closed on a particular engine to connect recognized requirements, thus offering extreme pliability to organize different subsets of sources on the uniform physical engine to separate particular needs of 
provision demands. Moreover, by actively drifting virtual engines through physical engines, jobs can be united and unexploited sources can supplement a minimal-power level, organized to activate at minimum functioning points to keep energy or turned off.

c) Facility Allocator: Performs as the connection between clients and the Cloud frame. It involves the interface of the surveying modules to assistance energy-competent resource organization.

- Green Representative: Confers with the clients to settle the provision level agreements with specific rates and consequences between the clients and system provider depending on the client's quality of service (QoS) needs and energy protecting outlines.

- Facility Analyzer: Explores and clarifies the facility requests of a presented demand earlier determining whether to receive or discard it. Thus, it requires the newest stack and energy records from the virtual machine (VM) administrator and energy monitor correspondingly.

- Client Profile: Assembles particular features of clients to facilitate significant clients can be settled distinct benefits and arranged over other clients.

- Valuing: Selects how facility requirements are charged to achieve the source and request of computing supplies and simplify in arranging provision distributions efficiently.

- Energy Inspection: Perceives and resolves which physical engines to power off/on.

- Facility Scheduler: Consigns requirements to VMs and decides resource privileges for assigned VMs. Beside it chooses when VMs are to be separated or inserted to meet the requirement.

- VM Administrator: Maintains trace of the accessibility of VMs and resource privileges.

- Accounting: Preserves the authentic practice of sources by demands to compute management costs.

d) Clients: Cloud clients present facility needs from anyplace to the cloud. It is imperative to observe that there can be a change between clients and users of utilized facilities.

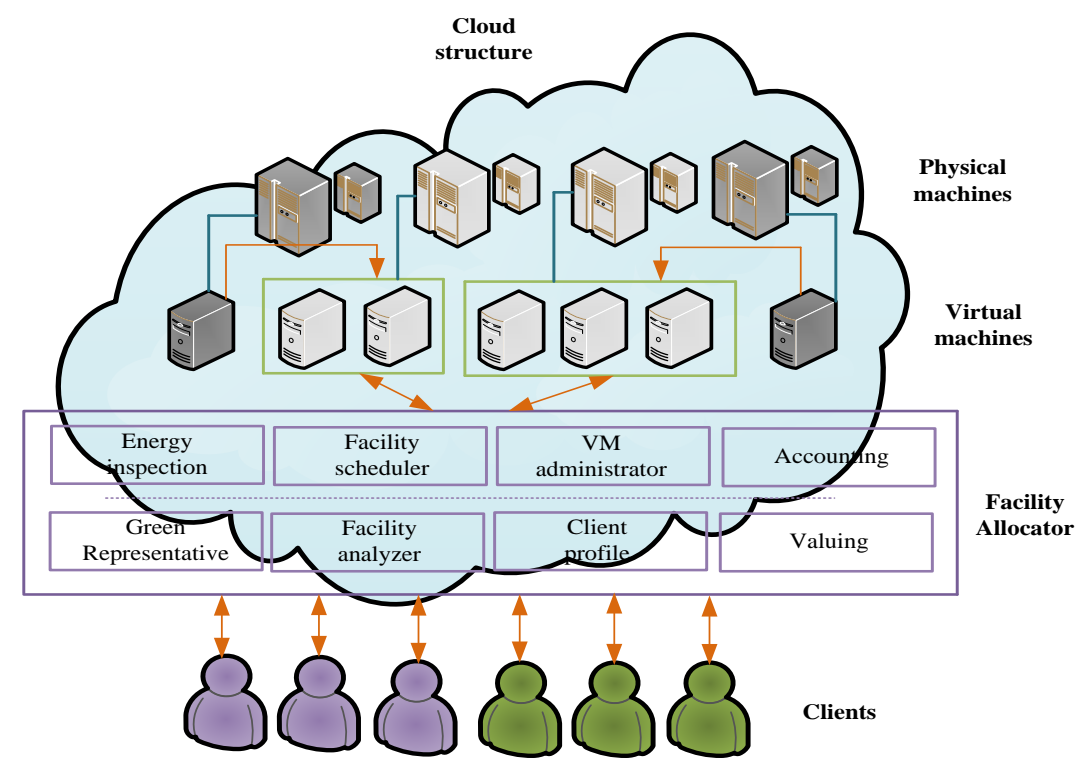

Figure 1. Architectural View of Cloud 


\section{Energy-Conscious Data Centre Resource Provision}

Energy expended by the data center can be generally considered into two sections specifically energy utilizes by apparatus like servers and utilize by foundation services like power systems. The energy volume expended by the subsections depend on the outline of the data center along with the efficacy of the apparatus. For instance, as per the data printed by the Infotech Company, the major energy user in a usual data center is the freezing structure (50\%) [8], whereas storage procedures and servers $(26 \%)$ position second in the energy utilization order. A method to achieve internet data center energy utilization contains some of four main stages: attribute extraction, model formation, confirmation, and prediction.

- Attribute Extraction: To diminish the energy utilization of the data center, it is important to determine the energy utilization of its constituents [9] and recognize where the maximum of the energy is consumed.

- Model Formation: The particular input structures are operated to develop an energy utilization paradigm by assessment procedures for example machine learning. Traditional assessment procedures may not generate precise outcomes in some states where machine learning methods may function well. The result of this stage is a power paradigm.

- Confirmation: Afterward, the paradigm needs to be authenticated for its suitability for its projected reasons.

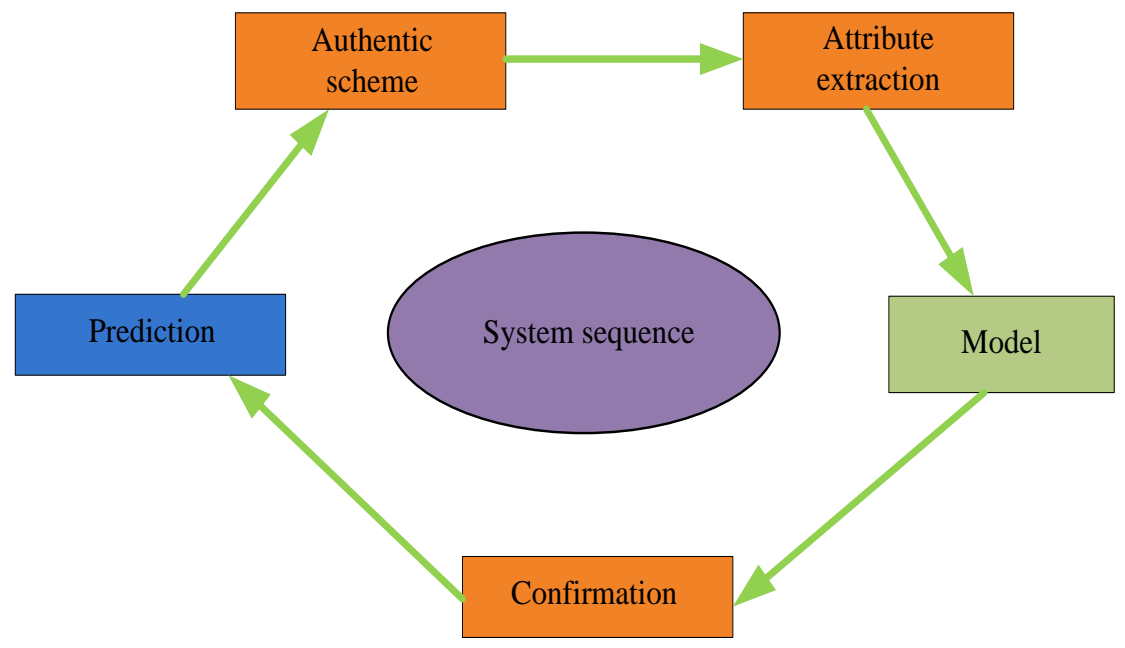

\section{Figure 2. An Organized Outlook of the Energy Utilization Forming and Forecast Procedure}

- Model Usage: The categorized paradigm can be operated as the base for forecasting the constituent energy utilization. Such forecasting can be operated to expand the energy efficacy of the data center, for instance by integrating the prototype into procedures like energy or temperature cognizant scheduling [10], active or dynamic voltage frequency scaling (DVFS) $[11,12]$ or expanding the algorithms used by the functions [13] to formulate data centers further energy effective.

A scheme for actively adjusting the energy utilization of a data center is ElasticTree [14]. It includes some logical components as routing, optimizer and 
power regulator as presented in Figure 3. The function of the optimizer is to locate the least power system subset that assures present traffic states. It returns a unit of dynamic modules to both the routing segments and power control. Routing selects tracks for all flows, afterward drives routes into the system, whereas power control switches the positions of linecards and complete switches.

The VM provision challenge can be split in two as an entrance of latest demands for VM facility and assigning the VMs on hosts, while the next segment is VMs optimization [15]. The leading segment is perceived as a bin arranging problem with variable bin scales and rates. To resolve it, Best Fit Decreasing procedure is applied that is pointed to use simply $11 / 9$ OPT +1 bins [16]. Optimization of VMs allocation is approved out in dual phases: at the initial phase choose VMs which need to be shifted, next selected VMs are positioned on hosts by MBFD algorithm.

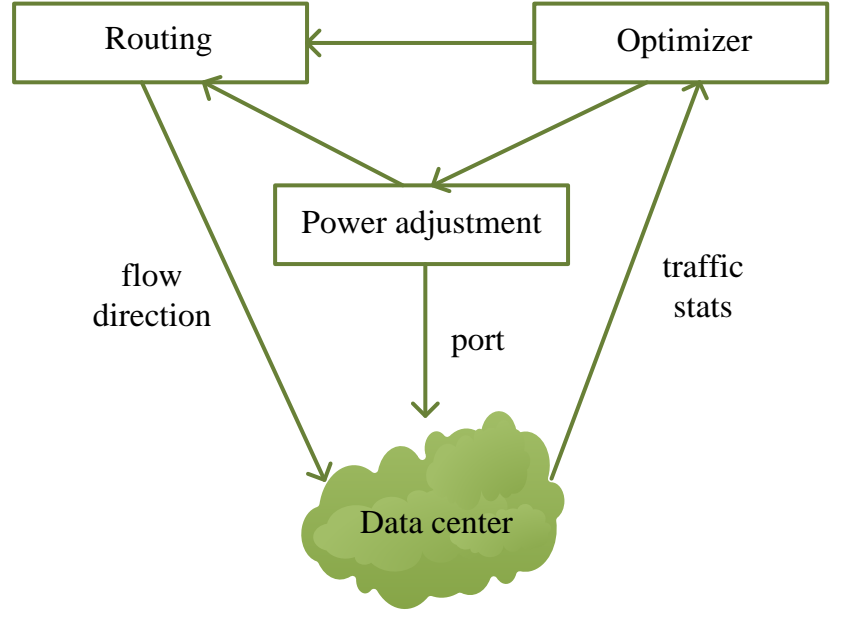

Figure 3. System Schema

Some heuristics for selecting VMs migration is proposed like single threshold which is founded on the perception of locating higher application threshold for hosts and locating VMs though fixing the whole application of CPU beneath the threshold. At every time form, all VMs are rearranged by MBFD with a supplementary circumstance of retaining the higher application threshold not interrupted. The latest service is attained by VMs live migration [17]. The additional heuristics are founded on the design of adjusting higher and lower application thresholds for hosts and retaining complete application of CPU between these thresholds in all VMs.

\section{Challenges of Regularity Resources}

In this section, some crucial difficulties which can be focused at the management level are categorized. Cloud system profoundly depend on virtualization technology which delivers the capacity to relocate VMs between nodes by offline migration. This permits the process of active association of VMs to a nominal figure of nodes along with existing resource supplies. Therefore, the inactive nodes can be changed off or set to an energy saving form to decrease absolute energy utilization by the data center. Even with the reserves of energy, an assertive association of VMs may direct to an operation outrage and, therefore outcome in service level agreements desecration. A current analysis reveals that internet data centers signify a huge and quickly rising energy utilization region of the economy and is a substantial cause of $\mathrm{CO} 2$ releases [18]. 


\subsection{Energy-concerned Active Resource Provision}

Current progress in virtualization has ensued in its propagation of practice through data centers. It allows active movement of VMs along with QoS prerequisites with supports the association of VMs between nodes. As VMs do not utilize total delivered resources, they can be rationally associated and resized on a nominal figure of nodes, though inactive nodes can be shifted off. Presently, resource provision in a data center points to specify extreme operational as contacting service level agreements, devoid of an attention on allotting VMs to reduce energy utilization. Several concerns as extreme power driving of a server may possibly lessen its consistency, revolving resources off in an active situation is perilous from a QoS potential and confirming service level agreements realizes questions to precise function implementation organization in virtualized situations are focused to survey both operation and energy competence.

\subsection{Quality of Service based Resource Specification}

The data center could deliver various stages of operation to the consumers; thus, QoS-responsive resource association shows a crucial part of cloud computing. Hence it is indispensable to perform an analysis of cloud facilities and capacities to recognize mutual activities, outlines and search load estimating methods that can possibly direct to additional proficient resource specification and resultant energy proficiency.

\subsection{Simulated Topologies Optimization}

VMs frequently connect each other to authenticate topologies in virtualized data centers. Though, because of non-optimized provision or VM movements, the interactive VMs may perhaps finish up hosted on rationally reserved nodes maintaining expensive data transmission between each other. The system interface may contain network adjustments that expend major volume of power if the interactive VMs are distributed to the hosts in separate frames. To eradicate this data transmission expenses and reduce power depletion, it is essential to perceive the contact between VMs and engage them on the uniform nodes.

\subsection{Thermal Conditions Optimization and Process of Cooling Scheme}

A substantial segment of electrical force utilized by figuring resources is changed into heat. Extreme heat directs to a number of difficulties, like lessened consistency and obtainability of the system, along with reduced lifespan of tools. To retain the modules in secure functioning temperature with avoiding failures and collisions, the released heat needs to degenerate. The cooling difficulty turns into particularly vital for latest servers which direct to an extreme mass of processing resources and unclear heat degeneracy. Some effort on forming data center's thermal topology can direct to new effective workload location [19].

\subsection{VMs Competent Alliance for Overseeing Heterogeneous Assignments}

Cloud structure provides consumers with the facility to provision simulated machines [20] and assigns any type of functions to them. This points to the event that separate sorts of functions like technical and business can be allotted on the single node. Though, it is not clear how functions can effect each other, as they possibly are records or network system so forming the constant load on the sources. Recent methods to VMs energy proficient alliance in data centers do not consider the difficulty of joining separate sorts of assignment. Typical ways generally effort on specific workload category or do not count separate sorts of efforts presuming 
identical workload. A smart association of VMs with distinctive workload categories is suggested in [15].

\section{Conclusion}

Energy-conserving technologies have the diverse influence of the energy conserving at separate levels of data centers from a universal viewpoint. Data centers are the strengths of presents cloud structures. This study presents a unique effort on the obtainable energy-conserving technologies for data centers. In future, there's a quantity of study that we can scheme to perform, which may perhaps progress the operation of data center along with GreenCloud and fetch compact worth to consumers to attain their corporate objectives and community concern in Green IT Thus, we assume scholars universal to place in a robust thrust on exposed encounters categorized in this survey to enrich energy-competent organization of cloud computing atmospheres.

\section{Acknowledgments}

The authors would like to thanks the anonymous reviewers for reviewing the manuscript.

\section{References}

[1] K. Kant, "Data center evolution: A tutorial on state of the art, issues, and challenges", Computer Networks, vol. 53, no. 17, (2009), pp. 2939-2965.

[2] A. Weiss, "Computing in the Clouds", netWorker, vol. 11, no. 4, (2007), pp. 16-25.

[3] M. Abdullah-Al-Shafi and A. N. Bahar, "Cloud Computing: An Aspect of Information System", International Journal of Applied Information Systems, vol. 10, no. 4, (2016), pp. 46-50.

[4] R. Buyya, C. S. Yeo and S. Venugop, "Market-oriented cloud computing: Vision, hype, and reality for delivering it services as computing utilities", In Proceedings of the 10th IEEE International Conference on High Performance Computing and Communications (HPCC-08, IEEE CS Press, Los Alamitos, CA, USA), (2008).

[5] M. Abdullah-Al-Shafi, A. N. Bahar and S. Saha, "Mobile On-demand Computing: The Future Generation of Cloud", International Journal of Future Generation Communication and Networking, vol. 9, no. 11, (2016), pp. 161-178.

[6] EPA Report on Server and Data Center Energy Efficiency U.S. Environmental. Protection Agency, ENERGY STAR Program, (2007).

[7] R. Raghavendra, P. Ranganathan, V. Talwar, Z. Wang and X. Zhu, "No Power Struggles: Coordinated multi-level power management for the data center", In Thirteenth International Conference on Architectural Support for Programming Languages and Operating Systems (ASPLOS '08), (2008).

[8] W. V. Heddeghem, W. S. Lambert, B. Lannoo, D. Colle, M. Pickavet and P. Demeester, "Trends in worldwide ICT electricity consumption from 2007 to 2012", Computer Communications, vol. 50, (2014), pp. 64-76.

[9] D. J. Brown and C. Reams, "Toward energy-efficient computing", Communications of the ACM, vol. 53, no. 3, (2010), pp. 50-58.

[10] F. Bellosa, "The benefits of event: Driven energy accounting in powersensitive systems", Proc. 9th Workshop ACM SIGOPS EQ-Beyond PC-New Challenges Oper. Syst., (2000), pp. 37-42.

[11] Y. Hotta, M. Sato, H. Kimura, S. Matsuoka, T. Boku and D, Takahashi, "Profile-based optimization of power performance by using dynamic voltage scaling on a PC cluster”, Proc. 20th IPDPS, (2006), pp. 18.

[12] M. Weiser, B. Welch, A. Demers and S. Shenker, "Scheduling for reduced CPU energy", Proc. 1st USENIX Conf. OSDI, (1994), pp. 1-11.

[13] A. Beloglazov and R. Buyya, "Energy efficient resource management in virtualized cloud data centers", Proc. 10th IEEE/ACM Int. CCGrid, (2010), pp. 826-831.

[14] B. Heller, S. Seetharaman, P. Mahadevan, Y. Yiakoumis, P. Sharma, S. Banerjee and N. McKeown, "ElasticTree: Saving Energy in Data Center Networks", In NSDI, vol. 10, (2010), pp. 249-264.

[15] R. Buyya, A. Beloglazov and J. Abawajy, "Energy-efficient management of data center resources for cloud computing: A vision, architectural elements, and open challenges", arXiv preprint arXiv:1006.0308, (2010).

[16] M. Yue, "A simple proof of the inequality FFD (L)< 11/9 OPT (L)+ 1, for all 1 for the FFD bin-packing algorithm”, Acta Mathematicae Applicatae Sinica (English Series), vol. 7, no. 4, (1991), pp. 321-331. 
[17] C. Clark, K. Fraser, S. Hand, J. G. Hansen, E. Jul, C. Limpach, I. Pratt and A. Warfield, "Live migration of virtual machines", In Proceedings of the 2nd Symposium on Networked Systems Design and Implementation (NSDI'05). USENIX, (2005).

[18] P. Johnson and T. Marker, "Data centre energy efficiency product profile", Pitt \& Sherry, report to equipment energy efficiency committee (E3) of The Australian Government Department of the Environment, Water, Heritage and the Arts (DEWHA), (2009).

[19] J. Moore, J. Chase and P. Ranganathan, "Weatherman: Automated, online, and predictive thermal mapping and management for data center", In Proc. of the International Conference on Autonomic Computing, (2006).

[20] A. N. Bahar, M. A. Habib and M. M. Islam, "Security architecture for mobile cloud computing", International Journal of Scientific Knowledge Computing and Information Technology, vol. 3, no. 3, (2013), pp. 11-17.

[21] M. A. Laurenzano, M. Meswani, L. Carrington, A. Snavely, M. M. Tikir and S. Poole, "Reducing energy usage with memory and computation-aware dynamic frequency scaling", In European Conference on Parallel Processing, Springer, Berlin, Heidelberg, (2011), pp. 79-90.

[22] G. Sun, Y. Joo, Y. Chen, Y. Chen and Y. Xie, "A hybrid solid-state storage architecture for the performance, energy consumption, and lifetime improvement", In Emerging Memory Technologies, Springer, New York, (2014), pp. 51-77.

[23] M. Nijim, A. Manzanares and X. Qin, "An adaptive energy-conserving strategy for parallel disk systems", In Proceedings of the 12th IEEE/ACM International Symposium on Distributed Simulation and Real-Time Applications, IEEE Computer Society, (2008), pp. 75-82.

[24] M. A. Z. Alves, K. Khubaib, E. Ebrahimi, V. T. Narasiman, C. Villavieja, P. O. A. Navaux and Y. N. Patt, "Energy savings via dead sub-block prediction", In Computer Architecture and High Performance Computing (SBAC-PAD), 24th International Symposium on IEEE, (2012), pp. 51-58.

[25] S. Mittal, Z. Zhang and Y. Cao, "CASHIER: A cache energy saving technique for QoS systems", In 26th International Conference on VLSI Design, (2013), pp. 43-48.

\section{Authors}

Md. Abdullah-Al-Shafi received his B.Sc. (Engineering) in 2015 and Masters in 2018 from Institute of Information Technology (IIT), University of Dhaka, Bangladesh. He has more than 20 research article in world-renowned international journals and conference. His research area includes Distributed Computing, Quantum-dot Cellular Automation, Intelligent System and Wireless Sensor Network.

https://www.researchgate.net/profile/Abdullah_Al-Shafi

Ali Newaz Bahar received his M.Sc. (Engineering) in 2015. He is currently an Assistant Professor in the Department of Information and Communication Technology of Mawlana Bhashani Science and Technology University. His research area includes Quantum-dot Cellular Automation, Thin Film Solar Cell, Big Data Analysis, WSN and Fuzzy Set. https://www.researchgate.net/profile/Ali_Bahar5. 